\title{
Analysis about the influence of English Science News to the College English Study of College Students of Science Major Chenpeng Gao ${ }^{1, a}$, Yan Yang ${ }^{2, b, *}$
}

${ }^{1}$ Foreign Language Teaching and Researching Department, Hebei Univeristy, No. 180 East Wusi Road, Post code: 071002, Baoding City, Hebei Province, China.

${ }^{2}$ Foreign Language Teaching and Researching Department, Hebei Univeristy, No. 180 East Wusi Road, Post code: 071002, Baoding City, Hebei Province, China.y

agaochenpeng@126.com, b22520545@qq.com

${ }^{*}$ Corresponding author

\begin{abstract}
With the development and implement of National College English Reform, college students gradually have higher requirements to college English teaching and learning. This paper is based on the study of the learning process of 37 undergraduates majored in high-polymer of the author' university. Teachers apply video clips and text of English science and technology news during the learning and teaching process, analyze the consequence and influence of this learning method.
\end{abstract}

Keywords: English science and technology news, Undergraduates majored in science, College English learning.

\section{英语科技新闻对理科专业大学生大学英语学习的影响分析 一一河北大学2012级高分子专业学生为例 高辰鹏 ${ }^{1,} \mathrm{a}^{*}$, 杨艳 ${ }^{2, b}$, \\ ${ }^{1}$ 河北大学大学外语教研部, 北市区五四东路 180 号, 保定市, 河北省, 中国 2 河北大学大学外语教研部, 北市区五四东路 180 号, 保定市, 河北省, 中国 agaochenpeng@126.com, b22520545@qq.com \\ “通讯作者}

中文摘要: 随着国内大学英语教学改革的推 进与实施, 大学生对于大学应教学有了新的 要求。本篇论文以作者在所在学校教授的 37 名高分子专业学生为例, 通过在大学英语的 学习过程中添加对英语科技新闻视频与文
字的研习与使用, 以观察这种学习方法对于 理科专业大学生的英语学习效果的影响。

关键词: 英语科技新闻; 理科专业大学生; 大 学英语学习 


\section{1. 引言}

2004年开始, 教育部颁布《大学英语课 程教学要求》, 提出切实提高大学生英语综 合应用能力, 特别是听说能力的精神。大学 英语教学改革开展十年来取得了卓越的成 就, 但是与此同时, 大学生对于英语学习也 有了新的要求和目标。英国语言学家Peter. Strevens 做为 ESP (English for Special Purpose）方面的权威, 早在上世纪就提出 ESP教学带有四个根本性特征和两个可变 性特征（1988）。在其描述的四个根本性特 征中的第一点（必须满足学习者的特定需 求）、第三点（在句法、词汇、语篇、语义 以及语篇分析上, 重点必须放在那些与特定 专业、职业及与之相关的活动的语言运用 上) 和第四点 (与普通英语 (English For General Purposes）形成对照）上，均揭示了 未来英语教学的实用性、专业性特点。

本论文的作者于 2012年开始教授其所 在大学的化学环境与工程学院的高分子专 业的37名同学的大学公共英语课程, 所使用 的教材是由外研社 2008 年出版的《新视野大 学英语, 第二版》 (New Horizon College English, Second Edition）, 分为读写译课程 与视听说课程两部分。在作者的教学授课过 程中, 作者刻意的加入了 $\mathrm{VOA}$ 慢速英语

(Voice Of America, Special English) 的科 技新闻视频与音频, 并在使用音频练习的时 候附加以音频原文, 来加强学生的听说能力 的提升。两年过去了, 现在作者针对这些学 生的学习效果做一个总结与分析, 以期待对 今后的教学方式有帮助。

\section{2. 英语科技新闻对于理工科大学生词汇学 习的帮助}

随着现代科技的发展, 英语语言也在经 历着巨大的变化, 尤其在科技英语领域, 越 来越多的新词开始出现。这种情况对于理工 科学生来说, 既是一种机遇也是一种挑战。 论文的这一部分重点放在英语科技新闻中 新词汇产生对于理工科学生英语词汇学习 的帮助研究和分析。

\section{1 英语科技新闻的特点}

英语科技新闻是描述科技领域新的进 展与新的技术出现等内容的英语新闻。在新 闻的用词方面同基础英语没有绝对的界限, 但英语科技新闻作为一种特殊的文体, 在其 形成过程中也充分运用英语构词法,利用现 有的语言材料, 采用合成、词缀、缩略等构 词方法来表达新的含义。

\section{2 英语科技新闻中新词的构构成方法}

2.2.1 合成词法。两个或两个以上独立 词素组合在一起而构成的一个新词被称之 为合成词, 这种构词法也被称之为合成构词 法。

合成构词法是英语中最早出现的一种 新词的构成方式, 同时也是最为常见的一种 新词构成方式。例如: earthshaking: 翻天 覆地的, fast-developing industry: 高速发展 的产业。

首字母缩写与短语也可以成为构成词 的一部分。例如: 首字母缩写: HIV, 即可 以表示HIV病毒, 也可以表示HIV阳性, HIV 感染或HIV抗体等。短语缩写: on-demand, 即可以表示视频点播或者准视频点播, 也可 以表示广告点播、比赛点播或者音视频点 播。

2.2.2 词缀合成法。词缀合成法是英语 学习者提高词汇量的一种很有效的方式。它 是通过在词根的基础上添加前缀或者后缀 来构成新的词汇。新词的意义与词根意义紧 密相关, 却又取决于与所携带的词根的意 义。

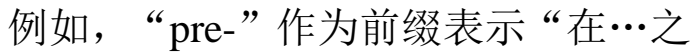
前”, 可以放在动词、名词和形容词之前, 可以携带或者不带连字符 “ - ”。例如 “prehistoric”史前的, “presuppose” 预先 假定。

2.2.3 广泛运用缩略词语。缩略语简单 易记,在科技英语中普遍运用。缩略词的出 现

例如: DNA (Deoxyribonucleic Acid) 脱氧核糖核酸, IC (Integrated Circuit) 集 成电路, CAD (Computer Aided Design) 计 算机辅助设计。 


\section{3 英语科技新闻对于理工科大学生词汇 学习的帮助的分析}

在接触到的英语科技新闻中, 层出不穷 的新词对于学习者来说既是一种机遇也是 一种挑战。理工科大学生对于新词的记忆和 使用具有一定的优势，其原因在于，首先， 在他们的专业课中也可以接触到许多新词, 中英文新词的对比可以使学习者在面对新 词的时候产生一种成就感, 从心理方面产生 优势, 从而帮助学生理解和记忆新词的意义 及用法。而通过学习各种构词法, 对于学习 者来说, 可以在面对新词的时候更好地理解 其意义, 并且, 由于对新词意义的获得往往 首先来自于自我揣测, 而后才是查询, 从而 使得学习者更容易对新词产生较为深刻的 印象。

\section{3. 英语科技新闻对于理工科大学生翻译技 巧的帮助}

\section{1 在作者所在学校里学习活动中的实际 应用。}

作者所教授的高分子专业的同学根据 所在学院的规定, 在毕业的时候被要求必须 翻译一遍英文科技文献。这一规定就对于学 生的翻译能力和水平提出了较高的要求。而 科技英语新闻音视频材料以及文本材料在 大学英语教学过程中的使用与练习, 恰恰提 供了这样一个机会给理工科的大学生们。

学生们由于从学院里得到了上述消息 的证实, 所以在平时的学习活动中也自愿的 投入时间和精力。学生学习自主性的提高, 既是源于学习考试的压力, 也是源于对英语 科技新闻的兴趣。

\section{2 在英语级别考试中的实际应用。}

随着2013年12月大学英语四六级考试 的再次改革, 考试中取消了句子与词汇的翻 译, 取而代之的是段落翻译。在段落翻译中 涉及的方面相当宽泛，包括有关中国节日、 历史事件、经济文化、旅游活动、社会发展 等各个方面。翻译题目中所出现的新词是许 多同学所从未或者很少接触过的。而对于长 期坚持进行英语科技新闻学习的同学来说, 首先具有的是心理方面的优势。
本篇论文的作者特意选择了本校化学 与环境工程学院中自己所教授的 37 名 2012 级高分子专业的学生进行调查, 在这些学生 中, 即将参加2014年6月14日下午进行的大 学英语六级考试的有 25 同学, 作者只选择他 们进行问卷调查, 并统计结果。

首先，在考试后，笔者通过在网络上搜 索和同学们的考试后反馈, 选择了 10 个在 2014年6月14日下午进行的大学英语六级考 试翻译部分中所出现的对于考生来说较为 陌生的新词作为调查内容:

另外, 由于本次考试采用的是一卷多题 的形式, 所以, 参加本次调查的学生所使用 的试卷也各不相同, 笔者在进行调查的时候 调查内容做了选择。在表 1 中 “人数”一项 中的 “分母” 表示使用试卷的人数, “分子” 表示答案基本准确的人数:

表1 2014年6月14日大学英语六级考试翻译试题中 新词翻译正确比率

\begin{tabular}{|c|c|c|c|}
\hline 选择词汇 & 参考翻译 & 人数 & 比率 \\
\hline $\begin{array}{l}\text { 沙尘暴和 } \\
\text { 建筑灰尘 }\end{array}$ & $\begin{array}{l}\text { sandstorms and } \\
\text { construction dust }\end{array}$ & $13 / 16$ & $81 \%$ \\
\hline 违章建筑 & $\begin{array}{c}\text { illegal } \\
\text { construction }\end{array}$ & $12 / 16$ & $75 \%$ \\
\hline $\begin{array}{l}\text { 垃圾处理 } \\
\text { 和污水处 } \\
\text { 理设施 }\end{array}$ & $\begin{array}{l}\text { he facilities of } \\
\text { municipal waste } \\
\text { treatment and } \\
\text { sewage treatment }\end{array}$ & $6 / 16$ & $37 \%$ \\
\hline 新粒子 & new particle & $1 / 5$ & $20 \%$ \\
\hline $\begin{array}{c}\text { 可持续战 } \\
\text { 略 }\end{array}$ & $\begin{array}{c}\text { sustainable } \\
\text { strategy }\end{array}$ & $4 / 5$ & $80 \%$ \\
\hline 3D打印 & $3 \mathrm{D}$ printing & $5 / 5$ & $100 \%$ \\
\hline 人造器官 & artificial organ & $3 / 5$ & $60 \%$ \\
\hline 中文热词 & buzzwords & $0 / 5$ & $0 \%$ \\
\hline $\begin{array}{c}\text { 土豪和大 } \\
\text { 妈 }\end{array}$ & tuhao and dama & $5 / 5$ & $100 \%$ \\
\hline 金价下跌 & $\begin{array}{l}\text { price of gold } \\
\text { decreased }\end{array}$ & $5 / 5$ & $100 \%$ \\
\hline
\end{tabular}

通过表1中 “比率”一项, 不难看出, 将近两年的英语科技新闻在教学活动中的 应用, 使得参加本次六级考试学生在面对试 卷中中科技新词的翻译中表现还是令人满 意的。而在一些流行词汇的翻译中 (最后两 个词汇），学生们更是表现出色，这源于他 
们平时对于时尚潮流英语的关注与兴趣投 入。

由此可以看出, 英语科技新闻在教学活 动中的应用对于英语级别考试中翻译部分 的应试效果的确有明显的帮助。这也对于学 生今后的学习方法和策略有很大的帮助和 鼓励作用。

\section{4. 英语科技新闻对于理工科大学生听力技 巧的帮助}

听力对于大多数大学英语的学习者来 说往往是考试中的一个难点, 这一看法在作 者所教授的高分子专业的学生中也得到了 他们的认可。所以在大学英语教学过程中, 作者利用课上时间对学生们进行听力练习, 同时, 在课下也给学生们预留了练习任务, 并在课上进行监督与检查, 以督促学生们按 照进度进行练习和提高。

\section{1 根据英语科技新闻特点选择适合理工 科大学生练习的音视频}

在英语科技新闻中, 过多的使用缩略 词, 所以在进行听力练习前, 有必要对学生 的听力习惯进行改正, 最好可以整理出一些 常见的缩略词以方便学生熟悉和记忆。或者 可以由教师在选择新闻篇章的时候, 提前把 篇章中所出现的缩略词挑拣出来, 并做解释 说明。

同时, 由于是科技新闻, 其中涉及到 的专用名词肯定会比较多, 而这些名词对于 学习者来说是相当迷茫的。这就有必要在选 择新闻篇章的时候可以选择一些内容难度 比较适合学生的篇章, 或者可以在听力练习 前把篇章中出现的新词提前挑选出来给学 生们注明, 或者在听力资料的播放中暂停下 来给学生们刻意的解释说明。

在作者的教学活动中, 作者采用的是 前两种方式。第三种方法由于会打断学生的 听力练习的连贯性, 而不建议采用。

\section{2 通过英语科技新闻的练习提高学生听 力技巧和水平}

新闻报道往往平铺直叙, 并没有太多 的转折承启, 所以在听的过程中只要集中精 力就会有所收获。但是依然有些学生在听力 练习过程中感到有些不适应, 因此作者特意 采用了以下的两个方法来指导学生:
第一, 在听力过程中尽可能的采用缩 写来记录自己听到内容。其中包括数字、时 间以及人名地名等可以简写缩写的内容。

第二，在科技新闻中，往往会出现大 量的事实、数据, 详尽的报到事件的来龙去 脉，因此新闻中的人名、地名以及组织单位 名称、时间、内容、目的、结果与原因才应 该引起学习者的注意。同时, 作者要求学生 自己在平时也要收集科技新闻的资料和相 关的背景知识, 以便在听力过程中可以不假 思索的记录下来。

另外, 在听完新闻之后, 作者要求学 生通过自己记录下来的内容, 用自己的话进 行复述, 这可以考察自己是不是听懂, 同时 还可以帮助记忆以及提高口语表达能力。这 对于理工科大学生来说, 既可以提高英语词 汇量的积累速度, 有利于翻译与写作, 又可 以提高听力水平, 同时还可以提高口语表达 能力, 可谓是一举多得。

\section{5. 结束语}

英语科技新闻以其流畅简洁的语言，丰 富多彩的知识范围, 时尚新潮的内容内涵, 逐渐的成为了英语学习者了解科技领域最 新进展的主要途径与方法。

把英语科技新闻引入大学英语教学课 堂教学, 为学生提高英语各项能力提供一定 的交际场景和题材, 为创新教育提供条件的 同时, 又为学生提供了接触世界最新科研成 果的机遇。就如同英国应用语言学研究的先 驱彼德・科德(S. Pit Corder，1918～1990) 所说的那样: 我们实际上不能 “教” 给学生 一门语言, 只能为学生创造一个适宜的语言 环境。

大学英语教师在授课的时候如果可以 对于英语科技新闻加以良好的利用, 既可以 在授课初步阶段良好的引入课本话题, 又可 以在授课过程中与学生更好地实现互动。对 于作者本校的理工科学生来说, 在毕业的时 候完成一篇英语科技论文的翻译又可以变 得轻松。同时, 如果理工科大学生可以在教 师的指导与帮助下, 对于英语科技新闻善加 利用, 那么, 他们自身英语各方面水平的提 高将指日可待。 


\section{References}

[1] SU Jie (Foreign Language Department of Xi'an University of Architecture and Technology, Xi'an Shaanxi 710055, China ) A. Banerjee, A joint economic-lot-size model for purchaser and vendor, Decision Sciences, vol.17, pp. 292-311, 1986.

[2] Lin, Fumei. Modern English Lexicology [M]. Anhui Education Press, 1985. (林福 美. 现代英语词汇学[M]. 安徽教育出版 社,1985.).

[3] Chen Yuanyuan. A Study on the Word Formation of EST Neologisms in CNN News English [M] 2006. （陈媛媛. 基于 $\mathrm{CNN}$ 科技新闻的科技新词构词分析）

[4] Lu, Guoqiang. Modern English Lexicology $[\mathrm{M}]$. Shanghai Foreign
Language Education Press,1983. （陆国 强.现代英语词汇学 $[\mathrm{M}]$.上海外语教育 出版社,1983.)

[5] S. Pit. Corder, Error Analysis and Interlanguage $[\mathrm{M}]$. Oxford: Oxford University Press. 1981.

[6] De Beaugrande, R. \& W. Dresseler. Introduction to Text Linguistics. 1981.Longdon: Longman

[7] Deng, Wei. On the Implicaiton of Informativity Theory of Texts to English News Listening [M].Journal of Huaihua University, 2008 (篇章的信息性在英语 新闻听力中的运用 $[\mathrm{M}]$. 怀化学院学报, 2008)

[8] He, Ying. Science News: Features and Translation Strategies. [M] Shanghai International Studies University, 2006. 